\title{
Effect of Surface Roughness on the Squeeze Film Characteristics of Circular Plates in the Presence of Conducting Couplestress Fluid and Transverse Magnetic Field
}

\author{
Syeda Tasneem Fathima, ${ }^{1}$ N. B. Naduvinamani, ${ }^{2}$ \\ J. Santhosh Kumar, ${ }^{1}$ and B. N. Hanumagowda ${ }^{3}$ \\ ${ }^{1}$ Department of Mathematics, Reva Institute of Technology and Management, Bangalore 560064, India \\ ${ }^{2}$ Tumkur University, Tumkur 572103, India \\ ${ }^{3}$ East Point College of Engineering, Bangalore 560049, India \\ Correspondence should be addressed to Syeda Tasneem Fathima; stasneem@revainstitution.org
}

Received 28 August 2014; Revised 12 November 2014; Accepted 17 December 2014

Academic Editor: Patrick De Baets

Copyright (C) 2015 Syeda Tasneem Fathima et al. This is an open access article distributed under the Creative Commons Attribution License, which permits unrestricted use, distribution, and reproduction in any medium, provided the original work is properly cited.

The combined effect of surface roughness and magnetic field on the performance characteristic of the circular plates lubricated with conducting couplestress fluid (CCSF) has been studied. On the basis of the Christensen Stochastic model, the generalized stochastic Reynold's equation is derived. Modified equations for the nondimensional pressure, load load-carrying capacity, and squeeze film time are derived. The results are presented both numerically and graphically and compared with conducting smooth surface case. It is observed that the surface roughness effects are more pronounced for couplestresses as compared to nonconducting Newtonian fluid (NCNF) in the presence of magnetic field.

\section{Introduction}

In recent years, the characteristics of Magnetohydrodynamics (MHD) in flow analysis are important for many engineering and industrial applications. The MHD bearings with conducting fluids possess the high thermal-conductivity and high electrical-conductivity features over the conventional bearings. The squeeze film lubrication for different configuration of bearings under the action of transverse magnetic field has been discussed by several authors [1-6]. Stochastic model for hydrodynamic lubrication of rough surfaces is studied by Christensen [7]. The combined effect of MHD and surface roughness has been studied by many authors [8-11] and it is found that effect of surface roughness in the presence of transverse magnetic field is significant on the squeeze film characteristics of the bearings.

It is known that the theory of couplestress fluid by Stokes [12] is a generalization of viscous fluid theory with couplestresses and body couples. Couplestress fluids are consequence of the assumption that the interaction of one part of the body on another across a surface is equivalent to a force and momentum distribution. It consists of rigid randomly oriented particles suspended in a viscous medium such as electrorheological fluids and synthetic fluids. Many studies have investigated the effect of couplestress on the squeeze film lubrication in the presence of transverse magnetic field [13-15]. The MHD bearings with conducting fluids possess numerous advantages over the conventional bearings.

Recently, Lin et al., [16] studied hydromagnetic nonNewtonian cylindrical squeeze film and its application to circular plates by derivation of modified lubrication equation. They found that the improved characteristics are further emphasized for circular plates operating with a larger magnetic field parameter and non-Newtonian parameter.

The effect of surface roughness on MHD conducting couplestress squeeze-film characteristics between the circular plates has not been studied so far. Hence, in this paper, an attempt has been made to study the combined effect of surface roughness and conducting couplestresses on the MHD squeeze-film characteristics between circular plates. 


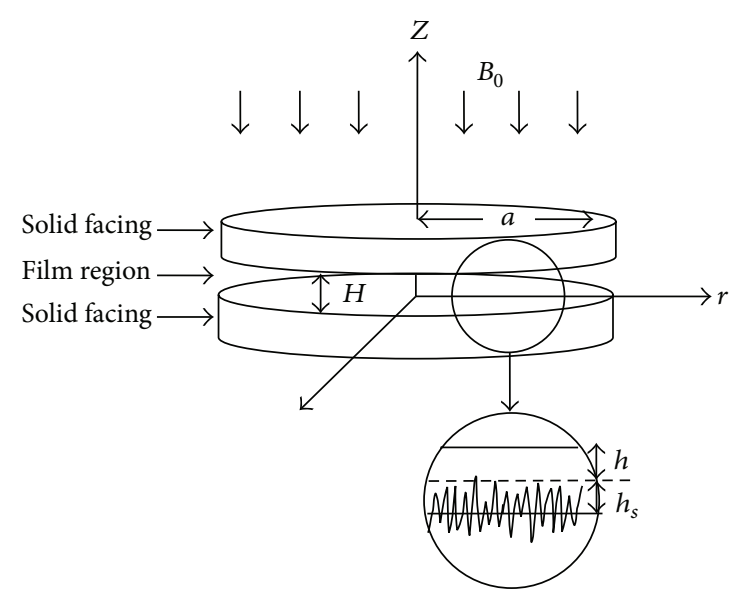

FIGURE 1: The physical configuration of rough circular plates in the presence of transverse magnetic field.

Expressions for the MHD squeeze-film pressure, load carrying capacity, and the time height relation are obtained. Results are compared numerically with the corresponding conventional case for different values Hartmann number $M_{0}$ studied by Lin et al., [16].

\section{Mathematical Formulation of the Problem}

Consider a squeezing flow between two rough circular plates approaching each other with squeezing velocity $V$ (= $d H / d t$ ), where $H$ is the film thickness between the two plates, $p$ is the film pressure, $u$ and $w$ are the velocity components, $\mu$ is the lubricant viscosity, $\eta$ is material constant responsible for couplestresses, and $\sigma$ is the conductivity of the lubricant. A uniform transverse magnetic field $B_{0}$ is applied to the bearing in the $Z$-direction as shown in Figure 1. It is assumed that the fluid film is thin and the body forces and the body couples are negligible. The third term in (1) is a Lorentz body force coming from $J_{y} B_{0}$ under the assumption that the induced magnetic field is much less than the applied magnetic field as described by Kuzma [17]. Then, from Ohm's law, the axial current density becomes $J_{y}=\sigma B_{0} u$. Therefore, the term $\sigma B_{0}^{2} u$ appears in (1).

Under the usual assumption of hydromagnetic lubrication theory applicable to thin films and Stokes theory for couplestresses, the continuity equation and the Magnetohydrodynamic (MHD) momentum equations in polar form becomes

$$
\begin{gathered}
\frac{\partial^{2} u}{\partial z^{2}}-\frac{\eta}{\mu} \frac{\partial^{4} u}{\partial z^{4}}-\frac{M_{0}^{2} u}{h_{0}^{2}}=\frac{1}{\mu} \frac{\partial p}{\partial r}, \\
\frac{\partial p}{\partial z}=0 \\
\frac{1}{r} \frac{\partial}{\partial r}(r u)+\frac{\partial w}{\partial z}=0 .
\end{gathered}
$$

The relevant boundary conditions for the velocity components are as follows.
(1) At the lower surface, $z=0$

$$
\begin{gathered}
u=0 \text { (no slip) } \\
\frac{\partial^{2} u}{\partial z^{2}}=0 \text { (vanishing of couplestresses) } \\
w=0 .
\end{gathered}
$$

(2) At the upper surface, $z=H$

$$
\begin{gathered}
u=0 \text { (no slip), } \\
\frac{\partial^{2} u}{\partial z^{2}}=0 \text { (vanishing of couplestress), } \\
w=\frac{d H}{d t} \text { (squeezing velocity). }
\end{gathered}
$$

The expressions for the radial component can be obtained by solving (1) subject to boundary conditions ( $4 a$ ) to (5c) we get

$$
\begin{aligned}
u= & -\frac{h_{0}^{2}}{\mu M_{0}^{2}} \frac{\partial p}{\partial r} \\
& \cdot\left\{\frac { 1 } { ( A ^ { 2 } - B ^ { 2 } ) } \left(\frac{B^{2} \operatorname{Cosh}(A(2 z-H) / 2 l)}{\operatorname{Cosh}(A H / 2 l)}\right.\right. \\
& \left.\left.-\frac{A^{2} \operatorname{Cosh}(B(2 z-H) / 2 l)}{\operatorname{Cosh}(B H / 2 l)}\right)+1\right\},
\end{aligned}
$$

where $h_{0}$ is the initial film thickness, $l\left(=(\eta / \mu)^{1 / 2}\right)$ is the couplestress parameter characterizing the non-Newtonian effects, $M_{0}\left(=B_{0} h_{0}(\sigma / \mu)^{1 / 2}\right)$ is the Hartmann number measuring the strength of the magnetic field, and $A$ and $B$ are dimensional quantities that depend on $l$ and $M_{0}$. Consider

$$
\begin{aligned}
& A=\sqrt{\left(\frac{1+\sqrt{1-4 l^{2} M_{0}^{2} / h_{0}^{2}}}{2}\right)}, \\
& B=\sqrt{\left(\frac{1-\sqrt{1-4 l^{2} M_{0}^{2} / h_{0}^{2}}}{2}\right)} .
\end{aligned}
$$

The integral form of the continuity equation (3) across the film thickness is

$$
\int_{0}^{H}\left\{\frac{1}{r} \frac{\partial}{\partial r}(r u)\right\} d z=-\int_{0}^{H}\left(\frac{\partial w}{\partial z}\right) d z .
$$

Substituting the expression of $u$ and applying the velocity boundary conditions, one can derive the modified Reynolds equation after performing the integration:

$$
\frac{1}{r} \frac{\partial}{\partial r} r\left\{\frac{\partial p}{\partial r} f\left(H, l, M_{0}\right)\right\}=\frac{\mu}{h_{0}^{2}} \frac{d H}{d t}
$$


where

$$
\begin{aligned}
& f\left(H, l, M_{0}\right) \\
& \quad=\frac{1}{M_{0}^{2}}\left\{\frac{2 l}{A^{2}-B^{2}}\left(\frac{B^{2}}{A} \tanh \frac{A H}{2 l}-\frac{A^{2}}{B} \tanh \frac{B H}{2 l}\right)+H\right\} .
\end{aligned}
$$

Let $g\left(h_{s}\right)$ be the probability density function of the stochastic film thickness $h_{s}$.

Taking the stochastic average of (9) with respect to $g\left(h_{s}\right)$, the averaged modified Reynolds type equation is obtained in the form

$$
\frac{1}{r} \frac{\partial}{\partial r}\left[r \frac{\partial E(p)}{\partial r} E\left\{f\left(H, l, M_{0}\right)\right\}\right]=\frac{\mu}{h_{0}^{2}} \frac{d E(H)}{d t},
$$

where

$$
E(\cdot)=\int_{-\infty}^{\infty}(\cdot) g\left(h_{s}\right) d h_{s} .
$$

In accordance with Christensen [7], we assume that

$$
g\left(h_{s}\right)= \begin{cases}\frac{35}{32 c^{7}}\left(c^{2}-h_{s}^{2}\right)^{3}, & \text { where }-c<h_{s}<c \\ 0 & \text { elsewhere, }\end{cases}
$$

where $\bar{\sigma}=c / 3$ is the standard deviation.

In context of Christensen stochastic theory for the hydrodynamic lubrication of rough surfaces, two roughness patterns are considered, namely, radial roughness pattern and azimuthal roughness pattern.

2.1. Radial Roughness Pattern. For the one-dimensional radial roughness pattern, the roughness structure has the form of long, narrow ridges and valleys running in the radial direction (i.e., they are straight ridges and valleys passing through $z=0$ and $r=0$ ). In this case, the film thickness takes the form

$$
H=h(t)+h_{s}(\theta, \xi) .
$$

The stochastic modified Reynolds equation (11) takes the form

$$
\frac{1}{r} \frac{\partial}{\partial r} r\left\{\frac{\partial E(p)}{\partial r} E\left[f\left(H, l, M_{0}\right)\right]\right\}=\frac{\mu}{h_{0}^{2}} \frac{d E(H)}{d t} .
$$

2.2. Azimuthal Roughness Pattern. For the one-dimensional azimuthal roughness, the bearing surfaces have the form of long narrow ridges and valleys running in the $\theta$-direction (i.e., they are circular ridges and valleys on the flat plate that are concentric on $z=0$ and $r=0$ ). In this case, the film thickness assumes the form

$$
H=h(t)+h_{s}(r, \xi) .
$$

The modified Reynolds type equation (11) takes the form

$$
\frac{1}{r} \frac{\partial}{\partial r} r\left[\frac{\partial E(p)}{\partial r} \frac{1}{E\left\{1 / f\left(H, l, M_{0}\right)\right\}}\right]=\frac{\mu}{h_{0}^{2}} \frac{d E(H)}{d t} .
$$

For an axisymmetric case, these equations reduce to

$$
\frac{1}{r} \frac{\partial}{\partial r}\left[r \frac{\partial E(p)}{\partial r} G\left(H, l, M_{0}, c\right)\right]=\frac{\mu}{h_{0}^{2}} \frac{d h}{d t},
$$

where

$$
\begin{gathered}
G\left(H, l, M_{0}, c\right) \\
=\left\{\begin{array}{l}
E\left(f\left(H, l, M_{0}\right)\right) \\
{\left[E\left(\frac{1}{f\left(H, l, M_{0}\right)}\right)\right]^{-1} \quad \text { for radial roughness }}
\end{array}\right. \\
E\left(f\left(H, l, M_{0}\right)\right)=\frac{35}{32 c^{7}} \int_{-c}^{c} f\left(H, l, M_{0}\right)\left(c^{2}-h_{s}^{2}\right)^{3} d h_{s}, \\
E\left(\frac{1}{f\left(H, l, M_{0}, c\right)}\right)=\frac{35}{32 c^{7}} \int_{-c}^{c} \frac{\left(c^{2}-h_{s}^{2}\right)^{3}}{f\left(H, l, M_{0}\right)} d h_{s}, \\
\frac{1}{r} \frac{\partial}{\partial r}\left(r \frac{\partial E(p)}{\partial r}\right)=\frac{\mu d h / d t}{h_{0}^{2} G\left(H, l, M_{0}, c\right)} .
\end{gathered}
$$

The relevant boundary conditions for the pressure field are

$$
\begin{aligned}
& \left(\frac{\partial E(p)}{\partial r}\right)_{r=0}=0, \\
& {[E(p(r))]_{r=a}=0 .}
\end{aligned}
$$

Solving (22) using the boundary conditions (23a) and (23b) gives

$$
E(p)=-\frac{\mu d h / d t}{4 h_{0}^{2} G\left(H, l, M_{0}, c\right)}\left(a^{2}-r^{2}\right) .
$$

The dimensionless form of the film pressure is given by

$$
P^{*}=-\frac{E(p) h_{0}^{3}}{\mu(d h / d t) \pi a^{2}}=\frac{\left[1-r^{* 2}\right]}{4 \pi G^{*}\left(H^{*}, l^{*}, M_{0}, C\right)},
$$

where

$$
\begin{gathered}
l^{*}=\frac{2 l}{h_{0}}, \quad r^{*}=\frac{r}{a}, \quad H^{*}=\frac{H}{h_{0}}, \\
C=\frac{c}{h_{0}}, \quad h^{*}=\frac{h}{h_{0}}, \quad h_{s}^{*}=\frac{h_{s}}{h_{0}}, \\
A^{*}=\left\{\frac{1+\left(1-M_{0}^{2} l^{* 2}\right)^{1 / 2}}{2}\right\}^{1 / 2}, \\
B^{*}=\left\{\frac{1-\left(1-M_{0}^{2} l^{* 2}\right)^{1 / 2}}{2}\right\}^{1 / 2},
\end{gathered}
$$




$$
\begin{aligned}
& G^{*}\left(H^{*}, l^{*}, M_{0}, C\right) \\
& =\left\{\begin{array}{l}
E\left(F\left(H^{*}, l^{*}, M_{0}\right)\right) \\
E\left(\frac{1}{F\left(H^{*}, l^{*}, M_{0}\right)}\right)^{-1} \quad \text { for radial roughness }
\end{array}\right. \\
& F\left(H^{*}, l^{*}, M_{0}\right) \\
& =\frac{1}{M_{0}^{2}}\left\{\frac{l^{*}}{A^{* 2}-B^{* 2}}\right. \\
& \left.\quad \cdot\left(\frac{B^{* 2}}{A^{*}} \tanh \frac{A^{*} H^{*}}{l^{*}}-\frac{A^{* 2}}{B^{*}} \tanh \frac{B^{*} H^{*}}{l^{*}}\right)+H^{*}\right\} .
\end{aligned}
$$

The load carrying capacity of the squeeze film is obtained by integrating the pressure field over the area of the plate

$$
E(W)=2 \pi \int_{0}^{a} E(p) r d r=-\frac{\mu \pi d H / d t a^{4}}{8 h_{0}^{2} G\left(H, l, M_{0}, c\right)} .
$$

The nondimensional mean instantaneous load carrying capacity $W^{*}$ of the squeeze film is given by

$$
W^{*}=-\frac{E(W) h_{0}^{3}}{\mu(d H / d t) a^{4} \pi^{2}}=\frac{1}{8 \pi G^{*}\left(H^{*}, l^{*}, M_{0}, C\right)} .
$$

For the constant load $W$, the time required for the film thickness to decrease to the value $h_{1}$ at time $t_{1}$ can be obtained by integrating (29). Hence,

$$
\begin{gathered}
T=-\frac{t E(W) h_{0}^{2}}{\mu \pi^{2} a^{4}}=\frac{1}{8 \pi} \int_{1}^{h_{1}^{*}} \frac{d H^{*}}{G^{*}\left(H^{*}, l^{*}, M_{0}, C\right)}, \\
T=\frac{1}{8 \pi} \int_{1}^{h_{1}^{*}} \frac{1}{G^{*}\left(H^{*}, l^{*}, M_{0}, C\right)} d H^{*},
\end{gathered}
$$

where $h_{1}^{*}=h_{1} / h_{0}$.

\section{Results and Discussions}

The combined effect of surface roughness and MHD on the conducting couplestress fluid lubrication between circular plates is analysed on basis of Christensen's stochastic theory [7] for two types of roughness patterns. The squeeze film characteristics are analysed with respect to three nondimensional parameters, namely, the Hartmann number $M_{0}^{*}$, conducting couplestress parameter $l^{*}$, and the roughness parameter $C$.

Figure 2 depicts the variation of nondimensional mean pressure $P^{*}$ with the axial coordinate $r$ as a function of the roughness parameter $C$ with the parametric values $M_{0}=3$, $l^{*}=0.3$, and $H^{*}=0.4$ for both roughness patterns. It is observed that, at $C=0$, the radial roughness pattern coincides with the azimuthal roughness pattern. Further, the increase in $P^{*}$ is more pronounced with azimuthal roughness pattern as compared to radial roughness pattern. Figure 3 represents the variation of nondimensional load carrying capacity $W^{*}$ with $H^{*}$ for different values of $l^{*}$. It is observed that, with the increasing of values of $l^{*}$, there is significant

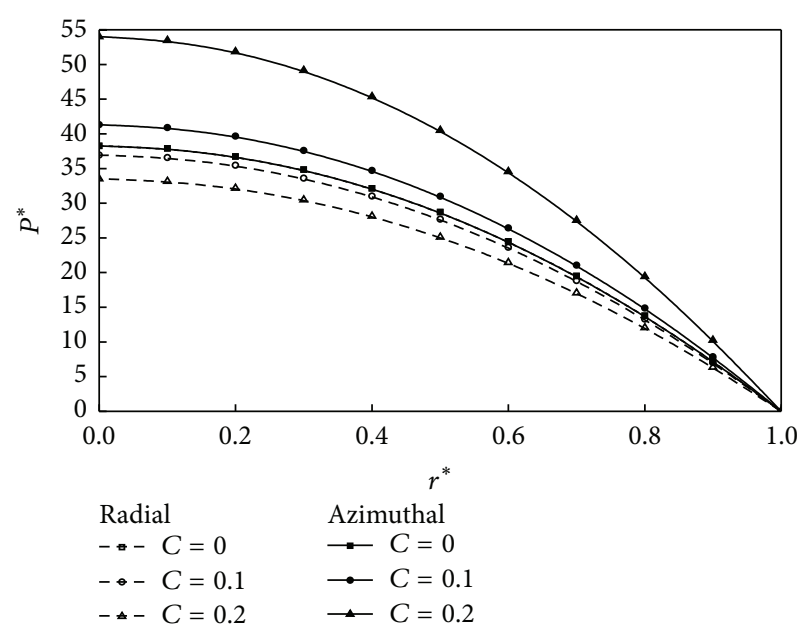

FIGURE 2: Variation of nondimensional pressure $P^{*}$ with $r^{*}$ for different values of $C$ with $M_{0}=3, l^{*}=0.3$, and $H^{*}=0.4$.

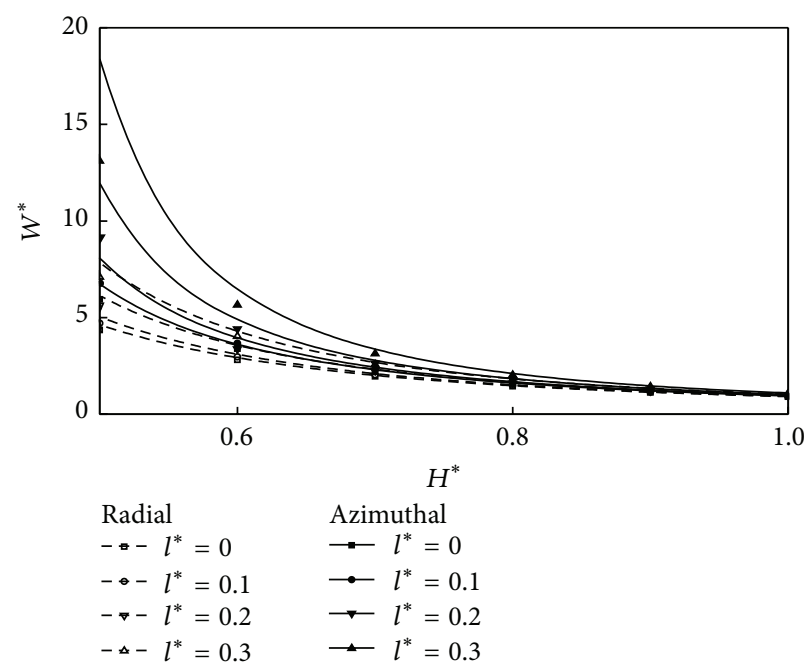

FIgURE 3: Variation of nondimensional load carrying capacity $W^{*}$ with $H^{*}$ for different values of $l^{*}$ with $M_{0}=3$ and $C=0.3$.

increase in the load carrying capacity $W^{*}$. The effects are more prominent for CCSF as compared to conventional NCNF. Figure 4 shows the variation of nondimensional mean load carrying capacity $W^{*}$ with $H^{*}$ for different values of $M_{0}$. It is seen that increasing effect of $M_{0}$ is to enhance the mean load carrying capacity for circular plates as compared to smooth plate case. Figure 5 shows the variation of nondimensional load $W^{*}$ with $H^{*}$ as a function of roughness parameter $C$ with $M_{0}=3$ and $l^{*}=0.3$. As seen from the figure that the load carrying capacity is significant with increasing values of roughness parameter and the effects are more prominent for azimuthal roughness pattern than those for radial roughness pattern. Variation on nondimensional squeeze film time $T^{*}$ with $h_{1}^{*}$ for different values of $l^{*}$ is shown in Figure 6. It is observed that, with the increasing of values of $l^{*}$, there is significant increase in squeeze film time $T^{*}$. The variation of response time $T^{*}$ as function $h_{1}^{*}$ is given in 


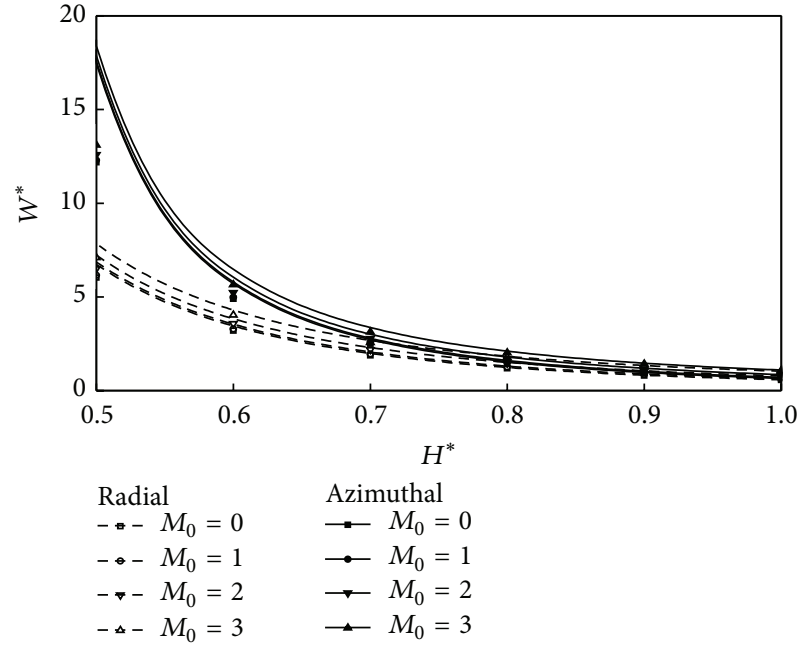

FIGURE 4: Variation of nondimensional load carrying capacity $W^{*}$ with $H^{*}$ for different values of $M_{0}$ with $l^{*}=0.3$ and $C=0.3$.

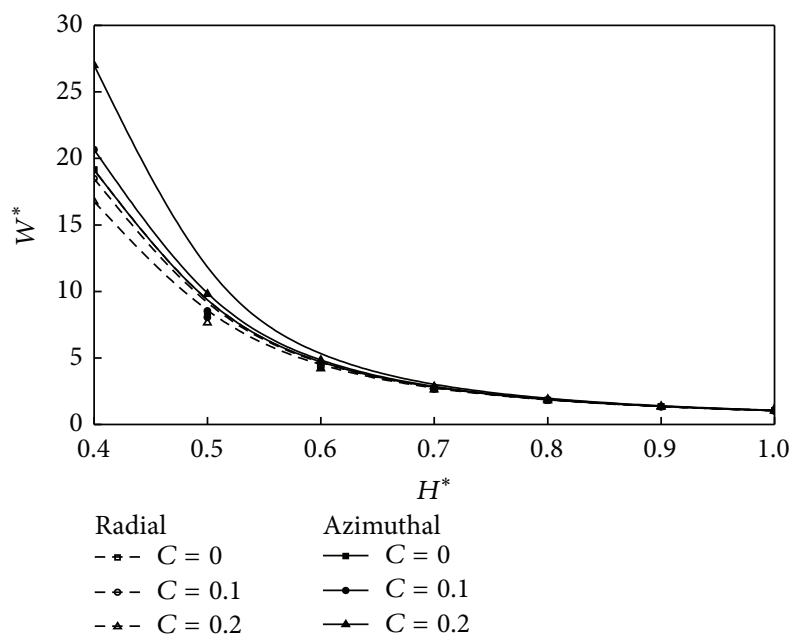

FIGURE 5: Variation of nondimensional load carrying capacity $W^{*}$ with $H^{*}$ for different values of $C$ with $M_{0}=3$ and $l^{*}=0.3$.

Figure 7 for various values of $M_{0}$. It is observed that the effect of magnetic parameter is to enhance the squeezing effect for rough circular plates as compared to smooth plates. Figure 8 shows the variation on nondimensional squeeze film time $T^{*}$ with $h_{1}^{*}$ for different values of $C$. It is observed that $T^{*}$ increases with increasing values of $C$. The effects are more pronounced for CCSF as compared to NCNF. It is observed that the effect of azimuthal/radial roughness patterns is to increase/decrease $T^{*}$ as compared to conventional case (i.e., $C=0)$. The time of approach is more pronounced for azimuthal roughness patterns as compared to the radial roughness pattern.

Table 1 shows the comparison between the previous analysis by Lin et al., [16] and the present analysis. It is observed from the table that there is significant increase in load and time for both the roughness patterns. An increase

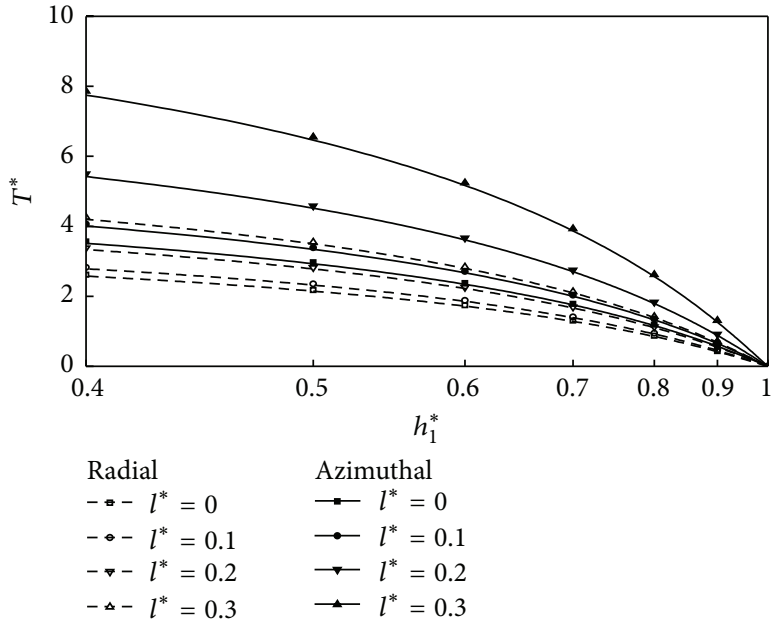

FIGURE 6: Variation of nondimensional load carrying capacity $T^{*}$ with $h_{1}^{*}$ for different values of $l^{*}$ with $M_{0}=3$ and $l^{*}=0.3$.

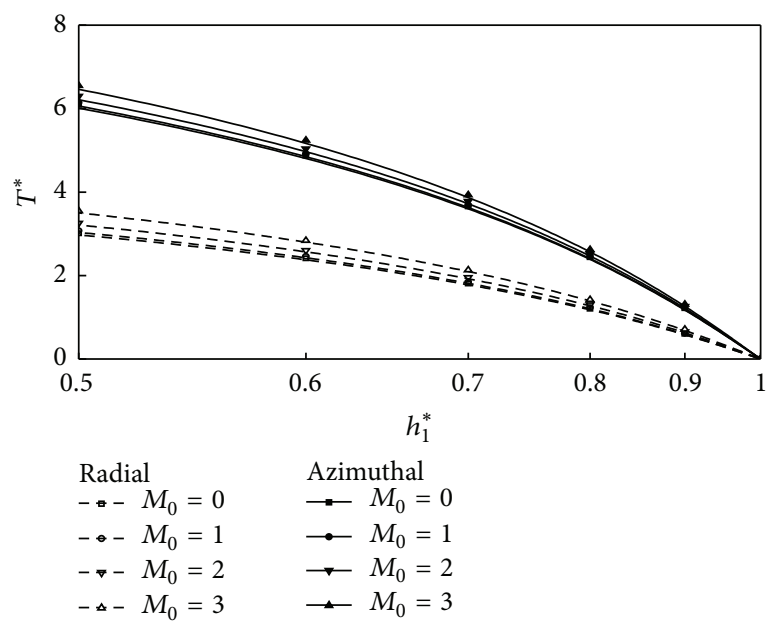

Figure 7: Variation of nondimensional load carrying capacity $T^{*}$ with $h_{1}^{*}$ for different values of $M_{0}$ with $l^{*}=0.3$ and $C=0.3$.

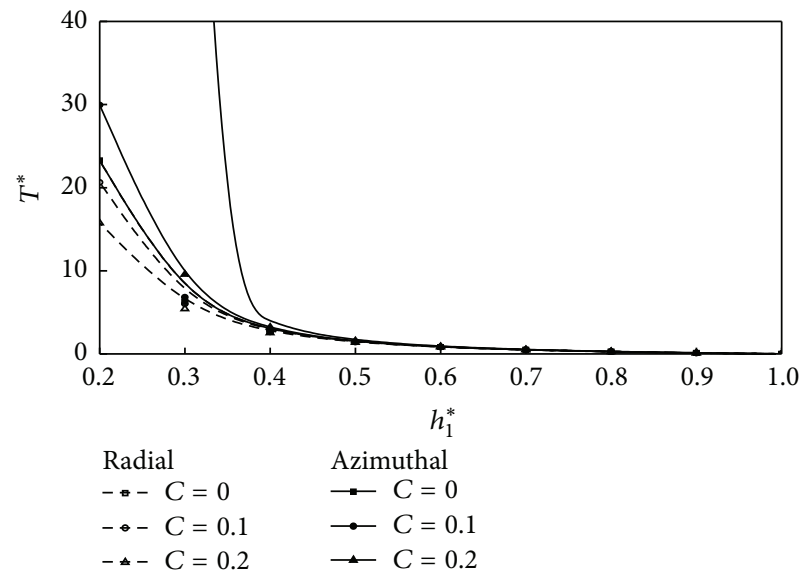

FIGURE 8: Variation of nondimensional squeeze film time $T^{*}$ with $h_{1}^{*}$ for different values of $C$ with $M_{0}=3$ and $l^{*}=0.3$. 
TABLE 1: Numerical comparison of load carrying capacity and the squeezing time between Lin et al., [16] and the present analysis with $H^{*}=0.4, l^{*}=0.3$, and $h_{1}^{*}=0.5$.

\begin{tabular}{|c|c|c|c|c|c|c|c|c|}
\hline \multirow{3}{*}{$M_{0}$} & \multicolumn{4}{|c|}{ Nondimensional load carrying capacity $W^{*}$} & \multicolumn{4}{|c|}{ Nondimensional squeezing time $T^{*}$} \\
\hline & \multirow{2}{*}{ Lin et al. [16] } & \multicolumn{3}{|c|}{ Present analysis } & \multirow{2}{*}{ Lin et al. [16] } & \multicolumn{3}{|c|}{ Present analysis } \\
\hline & & $C=0$ & $C=0.3$ (radial) & $C=0.3$ (azimuthal) & & $C=0$ & $C=0.3$ (radial) & $C=0.3$ (azimuthal) \\
\hline 0 & 18.0346 & 18.0345 & 13.068 & 51.1056 & 1.1262 & 1.1262 & 1.00394 & 1.51557 \\
\hline 1 & 18.1567 & 18.1566 & 13.2377 & 51.2369 & 1.15996 & 1.15996 & 1.04196 & 1.55012 \\
\hline 2 & 18.5232 & 18.5231 & 13.7416 & 51.6309 & 1.26118 & 1.26118 & 1.15448 & 1.65369 \\
\hline 3 & 19.1338 & 19.1338 & 14.5654 & 52.2874 & 1.42968 & 1.42968 & 1.33827 & 1.82611 \\
\hline
\end{tabular}

of $14.50 \% / 52.28 \%$ in $\mathrm{radial} / \mathrm{azimuthal}$ roughness pattern is observed for $M_{0}=3, C=0.3$.

\section{Conclusions}

The combined effect of surface roughness and MHD on the conducting couplestress squeeze-film characteristics between circular plates is presented on the basis of Christensen stochastic theory for rough surfaces [7]. From the above theoretical and numerical results, the following conclusions can be drawn.

(i) In the presence of a conducting couplestress fluid and externally applied transverse magnetic field, one-dimensional radial/azimuthal roughness patterns on the circular plates decrease/increase the loadcarrying capacity and response time as compared to the nonconducting case.

(ii) As the surface asperity increases, the large amount of load is delivered in the bearing and enhances the response time of squeeze-film motion as compared to the smooth case.

(iii) In the limiting case, as $C \rightarrow 0$, the result for both roughness patterns for one-dimensional circular plates can be reduced to smooth surface studied by Lin et al., [16] and the comparison is shown in Table 1.

\section{Nomenclature}

$a$ : Radius of the circular plates

$B_{0}$ : Applied magnetic field in the $z$-direction

c: Maximum asperity deviation from the nominal film height

$C$ : Dimensionless roughness parameter $\left(c / h_{0}\right)$

$H$ : Film thickness

$h_{0}$ : Initial film thickness

$h_{1}$ : Film thickness at time $t_{1}$

$h_{1}^{*}$ : Dimensionless film thickness after time $\Delta t$

$h_{s}$ : Stochastic film thickness

$M_{0}$ : Hartmann number $\left(=B_{0} h_{0}(\sigma / \mu)^{1 / 2}\right)$

$l$ : $\quad$ Couplestress parameter $(\eta / \mu)^{1 / 2}$

$l^{*}$ : Dimensionless couplestress parameter $2 l / h_{0}$ $r, \theta, z$ : Radial, angular, and axial coordinates

$u, v, w$ : Velocity components in film region

$E(P)$ : Pressure in the film region

$P^{*}: \quad$ Dimensionless pressure

$$
\left(=-E(p) h_{0}^{3} / \mu(d H / d t) \pi a^{2}\right)
$$

$t$ : Mean time of approach

$T^{*}$ : $\quad$ Dimensionless time of approach

$$
\left(=-t E(W) h_{0}^{2} / \mu \pi^{2} a^{4}\right)
$$

$V: \quad$ Squeezing velocity $(-d H / d t)$

$W^{*}$ : Dimensionless load carrying capacity

$$
\left(=-E(W) h_{0}^{3} / \mu(d H / d t) a^{4} \pi^{2}\right) \text {. }
$$

\section{Greek Symbols}

$\sigma:$ Conductivity of fluid

$\bar{\sigma}$ : Standard deviation

$\eta$ : Material constant responsible for couplestress

$\mu$ : Lubricant viscosity

$\xi$ : Random variable.

\section{Conflict of Interests}

The authors declare that there is no conflict of interests regarding the publication of this paper.

\section{References}

[1] W. F. Hughes, "The magnetohydrodynamic finite step slider bearing," Journal of Fluids Engingeering, vol. 85, no. 1, pp. 129$135,1963$.

[2] W. F. Hughes and R. A. Elco, "Magnetohydrodynamic lubrication flow between parallel rotating disks," Journal of Fluid Mechanics, vol. 13, no. 1, pp. 21-32, 1962.

[3] D. C. Huzma, "The Magneto-hydrodynamic parallel slider bearing," Transactions of the ASME Journal of Fluids Engineering. Series D, vol. 87, pp. 778-780, 1965.

[4] D. C. Kuzma, E. R. Maki, and R. J. Donnely, “The MHD squeeze film," Journal of Fluid Mechanics, vol. 19, no. 3, pp. 395-400, 1964.

[5] W. F. Huges and R. J. Elco, "Magnetohydrodynamic journal bearing," Journal of American Rocket Society, vol. 32, pp. 776778, 1962.

[6] J.-R. Lin, "Magneto-hydrodynamic squeeze film characteristics between annular disks," Industrial Lubrication and Tribology, vol. 53, no. 2, pp. 66-71, 2001. 
[7] H. Christensen, "Stochastic models for hydrodynamic lubrication of rough surfaces," Proceedings of the Institution of Mechanical Engineers, vol. 184, no. 55, pp. 1013-1026, 1969.

[8] N. M. Bujurke and R. B. Kudenatti, "MHD lubrication flow between rough rectangular plates," Fluid Dynamics Research, vol. 39, no. 4, pp. 334-345, 2007.

[9] N. B. Naduvinamani, S. T. Fathima, and S. Jamal, "Effect of roughness on hydromagnetic squeeze films between porous rectangular plates," Tribology International, vol. 43, no. 11, pp. 2145-2151, 2010.

[10] N. M. Bujurke, N. B. Naduvinamani, and D. P. Basti, "Effect of surface roughness on magnetohydrodynamic squeeze film characteristics between finite rectangular plates," Tribology International, vol. 44, no. 7-8, pp. 916-921, 2011.

[11] N. B. Naduvinamani and M. Rajashekar, "Effect of surface roughness on magneto-hydrodynamic squeeze-film characteristics between a sphere and a porous plane surface," Industrial Lubrication and Tribology, vol. 66, no. 3, pp. 365-372, 2014.

[12] V. K. Stokes, "Couple stresses in fluids," Physics of Fluids, vol. 9, no. 9, pp. 1709-1715, 1966.

[13] J.-R. Lin, L.-M. Chu, C.-R. Hung, and P.-Y. Wang, "Derivation of two-dimensional couple-stress hydromagnetic squeeze film Reynolds equation and application to wide parallel rectangular plates," Meccanica, vol. 48, no. 1, pp. 253-258, 2013.

[14] N. B. Naduvinamani, S. T. Fathima, and B. N. Hanumagowda, "Magneto-hydrodynamic couplestress squeeze film lubrication of circular stepped plates," Journal of Engineering Tribology, vol. 225, part I, pp. 111-119, 2011.

[15] S. T. Fathima, N. B. Naduvinamani, S. H. Marulappa, and H. Bannihalli, "A study on the performance of hydromagnetic squeeze film between anisotropic porous rectangular plates with couplestress fluids," Tribology Online, vol. 9, no. 1, pp. 1-9, 2014.

[16] J.-R. Lin, L.-M. Chu, L.-J. Liang, and P.-H. Lee, "Derivation of a modified lubrication equation for hydromagnetic nonNewtonian cylindrical squeeze films and its application to circular plates," Journal of Engineering Mathematics, vol. 77, pp. 69-75, 2012.

[17] D. C. Kuzma, "Magnetohydrodynamic squeeze films," Journal of Fluids Engineering, vol. 86, no. 3, pp. 441-444, 1964. 

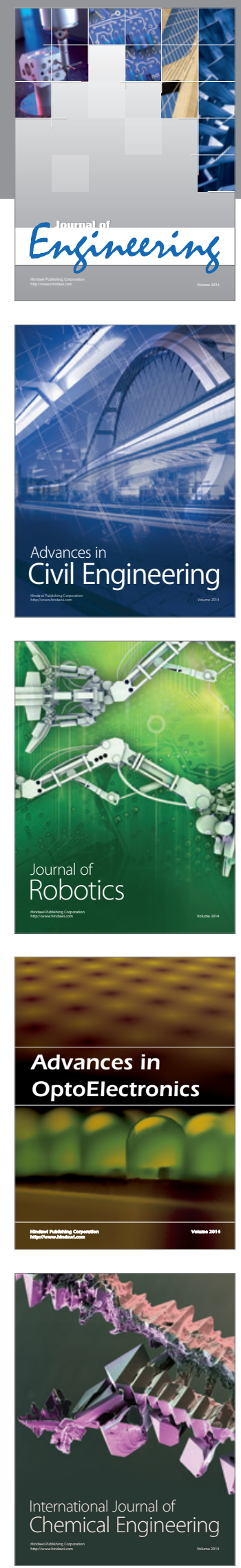

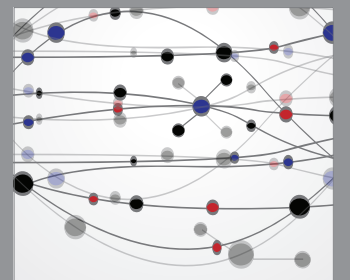

The Scientific World Journal
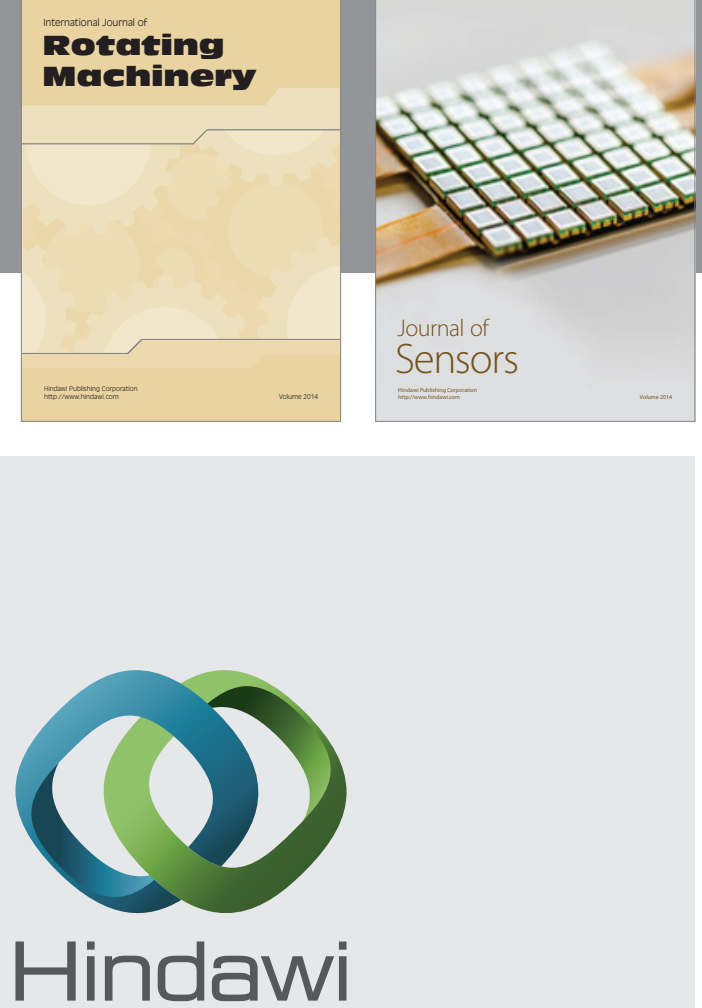

Submit your manuscripts at http://www.hindawi.com
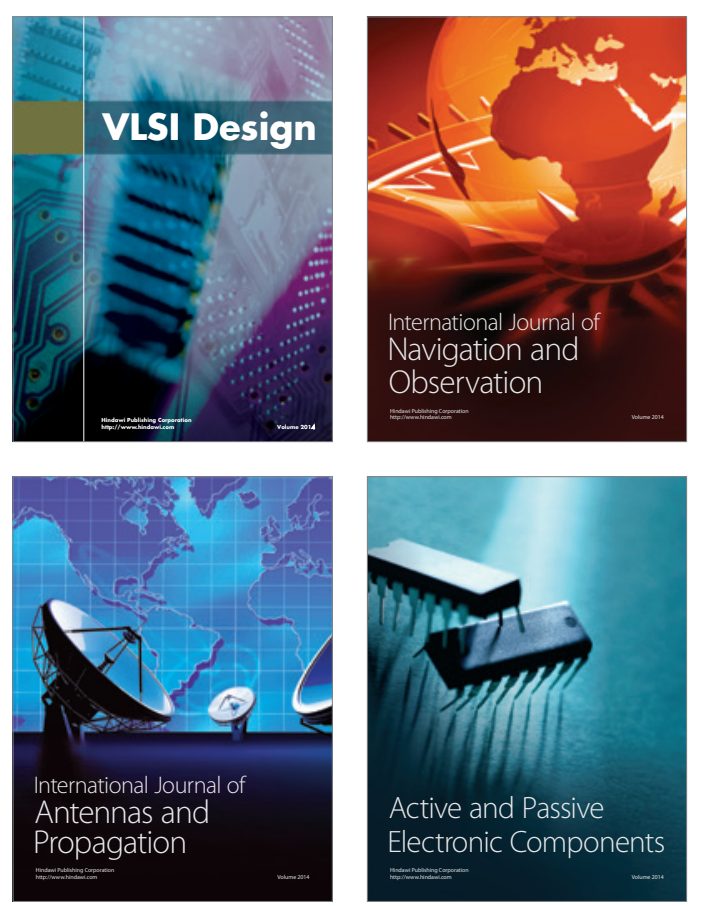
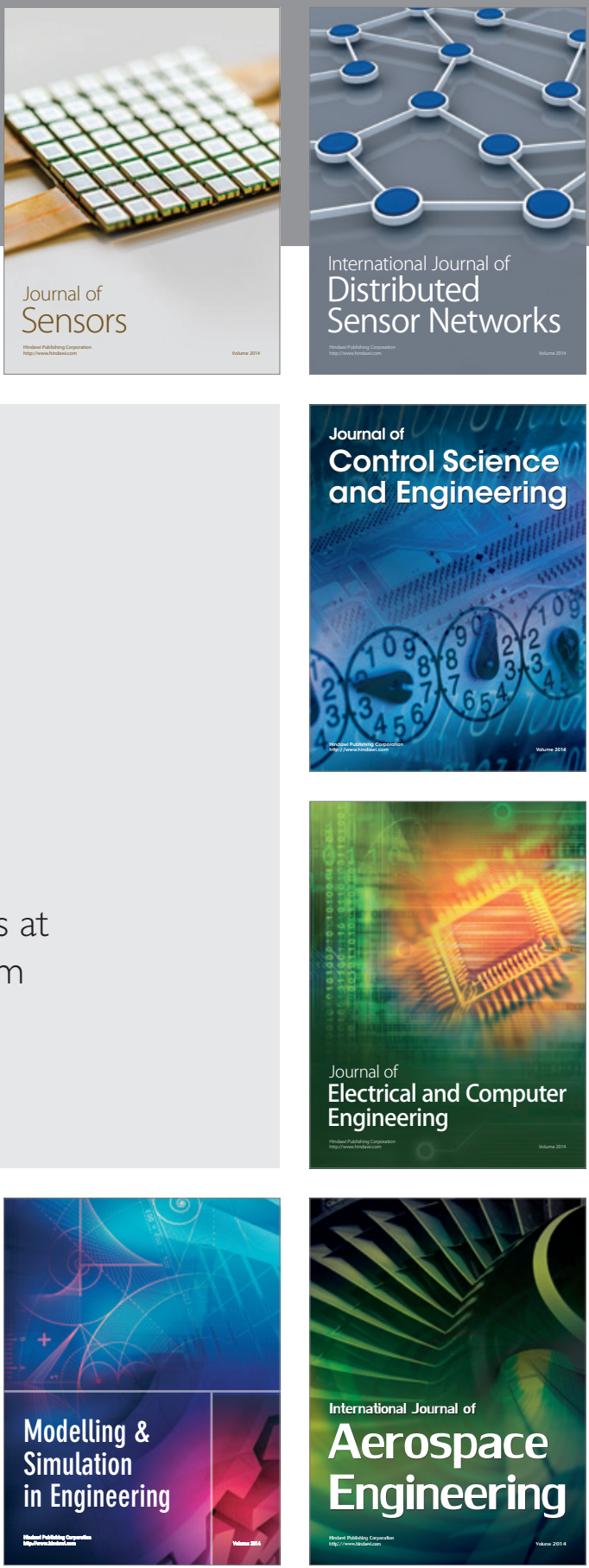

Journal of

Control Science

and Engineering
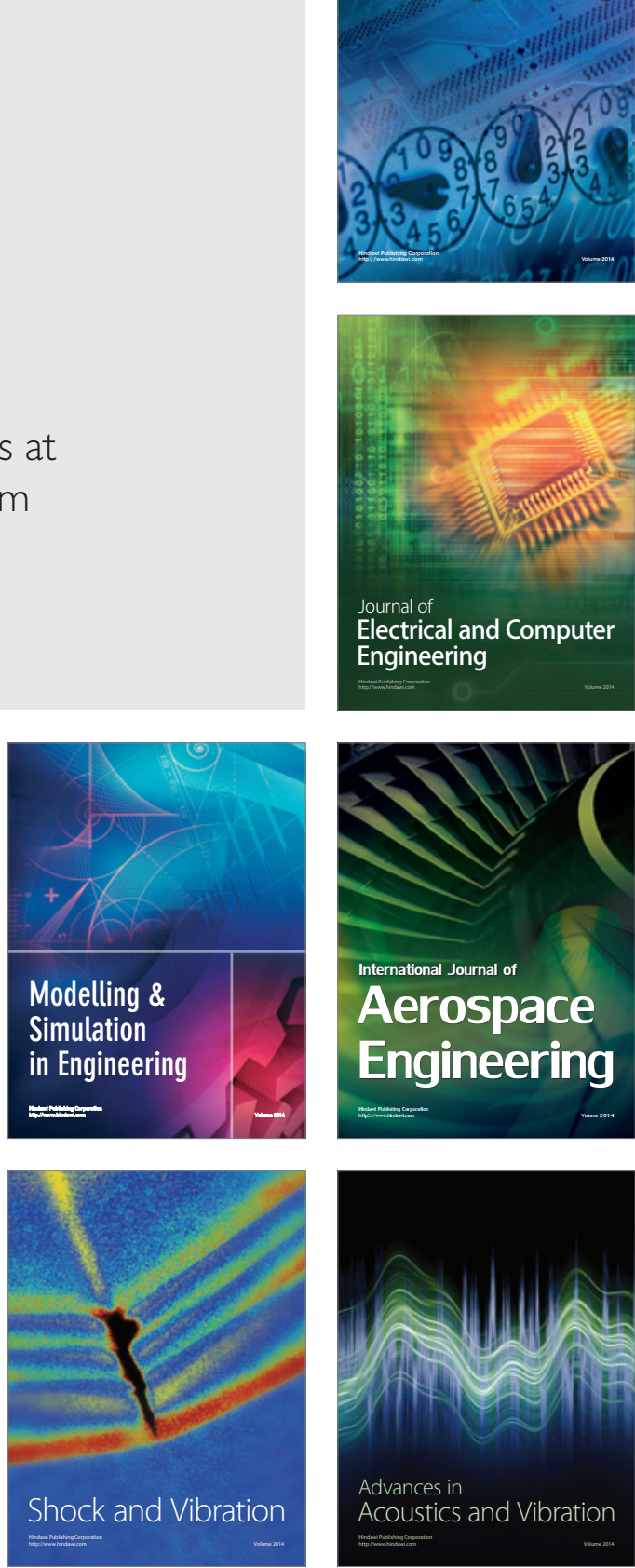